\title{
A Wireless Early Prediction System of Cardiac Arrest through fog enabled IoT
}

\author{
Deekshitha M R ${ }^{1}$, Karthik $S^{2}$, Lavanya $G^{3}$, Mohan Kumar $D^{4}$, Anusha K L \\ ${ }^{1}$ Student of EWIT, India, deekshithamraj@gmail.com \\ ${ }^{2}$ Student of EWIT, India, k3ksadhu@gmail.com \\ ${ }^{3}$ Student of EWIT,India,lavanyalakshmi1405@gmail.com \\ ${ }^{4}$ Student of EWIT,India,mohan.mohankumar41@gmail.com \\ ${ }^{5}$ Assistant Professor of EWIT, India, anushakl@ewit.edu
}

\begin{abstract}
The Objective of this paper is the appliance of a prototype model for monitoring the real time patient. This method makes use of physical parameters like pulse rate, body temperature, blood pressure, and ECG monitoring with the help of sensors. Currently there are number of techniques available to check the human health. In this new system, data is acquired by continuously monitoring the patient's health and is predicted by using Fog-Computing technique in the Raspberry-pi, and is transmitted using Wi-Fi module to the cloud. The inputs from patients are analyzed from the embedded processor and then the respective sensor values will be stored in the cloud. If the patient value have any abnormality then a manual notification message will be sent to the concerned person. And also the respective sensor values could be viewed by the registered concerned person under the availability of internet through the android app. This system is implemented by using the advanced IoT and the respective results are obtained.
\end{abstract}

Key words: BP, body temperature, ECG, Heart-rate, Cardiacarrest, FC technique.

\section{INTRODUCTION}

Health is very important and it is necessary to take care of the health. As the population is increasing, along with it health issues are also increasing due to pollution.

Wearable technologies popularity has been increased, with which the door for Internet of things (IOT) has opened one of the solution to healthcare. Worldwide, the average life expectancy of man was 100 years, but now it is 68 years 4 months for males and 72 years and 8 months for females, it has drastically reduced due to heart related disease and the cardiac arrest out-of-hospitals.

The new way of predicting the cardiac arrest earlier and using the wearable devices has opened a new way to increase the lifespan. Cardiac arrests in the elders are common and all the existing system predicts cardiac arrest in elders. It is estimated that on a yearly basis one-third to one-half of elder's population experiences the cardiac arrest[2]. Based on this the process is addressed by our research by focusing on ECG signals detection and analysis which eventually leads to the risk prediction.
As abnormal ECG patterns leads to cardiac arrest, our system alerts the user regarding a potential attack after the identification of an abnormal heart rate. In this society, cardiac arrest have huge aftermath as they cause tremendous concerns that are related to the decrease in the quality of life and the cost of healthcare has increased. Although many researches has been carried to find the automatic hearth attack detection, but the field of risk of early heart attack prediction is still lacks study and investigation. The need to identify all the fields that leads to heart attack is very challenging. Previously, seniors living all over the world were not adapting to the technologies when compared to younger generations. But, now it is changing that their entry into digital life is expanding. As researches say $59 \%$ of seniors report that they go online and $47 \%$ say they have a high-speed broadband connection at home. In addition, $77 \%$ of them have a phone and among that number, $18 \%$ are using smartphone devices[3,17].

With the increase in the processing capabilities of smartphones it can be provided with more number of sensors, GPS interfaces. Since smart phones are highly portable Smartphone based heart risk detection systems can function almost everywhere. Normally, integrated sensors and risk of injury caused due to cardiac arrest can detect by ECG. As a result of these benefits and because of the betterments in mobile technology, the elders may increase their smartphone use.

However, these existing heart attack detection system as disadvantage that it can only detect the risk only after its occurrence [4], following which the system sends an alarm to the caregivers. The elders may avoid injury from potential heart risk, if abnormal ECG patterns can be accurately identified using automated processes. The best way is to alert the users about their abnormal heart rate and bring to their attention that they are at a risk of a potential heart attack.

Hence our main focus is on early prediction of heart attack rather than its detection. The objective of this study is to develop a multisensory system that collects body temperature, heart rates, blood pressure, ECG. For this study we implemented an embedded sensory system with Wi-Fi module such that it will collect the ECG, body temperature, blood pressure and heart beat rate data using a smart phone in a common environment. our proposed system is not only useful for the elders, but also it has scope in identifying heart disease among adults, children and stroke patients, physical rehabilitation patients, and human behavior analysis research. 
Deekshitha M R et al., International Journal of Computing, Communications and Networking, 7(2) April - June 2018, 48-53

\section{A. Major Contributions}

In this paper, in order to predict the heart attack in advance we propose to use smartphones as the platform for developing the embedded system .Our major contributions are as follows:

- Developed a multisensory embedded IoT system for heart attack prediction.

- Proposed a smartphone based heart assistance system with a wearable ECG, temperature, blood pressure, heart beat sensor for elderly people as a novel application for predicting the cardiac arrest.

The rest of the paper is organized as follows: in the next section, we describe the relevant related work. In this section, we also discuss the difference between our system and the existing ones. Then we discuss the details of the proposed system. This section is followed by the evaluation of smartphone-based prototype system. Finally, we conclude the paper with some future research directions.

\section{RELATED WORK}

Identification of risk using wireless early prediction system of cardiac arrest using ECG patterns with the different embedded sensors has been a study of subject over the past History. In the previous approaches in case of predicting the cardiac arrest by utilizing the different kinds of embedded sensors like ECG, Heartbeat, Blood pressure and Body temperature which are attached to the subject's body for collecting data. But they have limited accuracy in case of predicting of cardiac arrest early.

Under the lots of circumstance that has been, a lot of work has been done to relate to it. It is necessary for us to learn about related/Existing system to see what aspects we can improve on. E-Health is been a very common topic now a days for research and many organization have taken the advantage of that EHealth by designing Advanced systems that connect patients with doctors around the world. "PatientsLikeMe" have launched its first online community in 2006 and its main goal was to listen to patients to identify their Health issues, outcome measures, symptoms, and treatments. That it is mainly focused on helping out the patients with E-Health related answer for their question: "Given patients status, what is the required best outcome that one can hope to achieve, and how it can be prevented by getting the solution. They answered patient questions in many different ways like cross checking the patients with similar related conditions which directly connects with each other and sharing their personal experiences with the doctors around the word.

Similarly "DailyStrength" is a social networking life everyone centered on support groups, where users provide emotional support for one another by discussing their struggles and successes with in their Daily Basis. This site contains online communities that deal with different types of medical conditions with the challenging life. Two major differences between "PatientsLikeMe" and "DailyStrength" is that, "DailyStrength" does not involve research institutes and does not have a mobile application. However, they are both global platforms that are very helpful for patients with health problems. "PatientsLikeMe" is more of a platform where patients share experiences of same symptoms while "DailyStrength" gives patients the option to talk to doctors.

Everyday IoT systems offers by producing content which are related to health and wellness of the Patients. Even though if there are many large number of Health service provider, none of them have the feature of hardware devices, that can be used to Monitor the patients HealthCare in their everyday activities and it notify them when it's emergence. Now a days there are lot of heart monitors out there that provide users to collect their ECG signals so they can keep track of their Health condition but none of those devices which who alert the users upon emergencies. "Qardiocore" is a very well-known and high quality heart monitor which is used to tracks a user's complete heart health and displays it through on smartphones. The device yields very accurate results and which is one of the best products in the market in terms of showing real time graphs of ECG and EKG. However, it only allows users to share data with their nearby doctors upon receiving to make a quick response in an offline manner, it does not give them the notification option of alerting them in real time when their patients is in the very serious condition and it certainly does not predict heart attacks. Neither of the above mentioned systems have the capability of predicting heart attack.

Later an embedded sensory system with a Low Energy(LE) Bluetooth communication module is implemented to collect ECG and body temperature data using a smartphone in a common environment as shown in Figure 1.

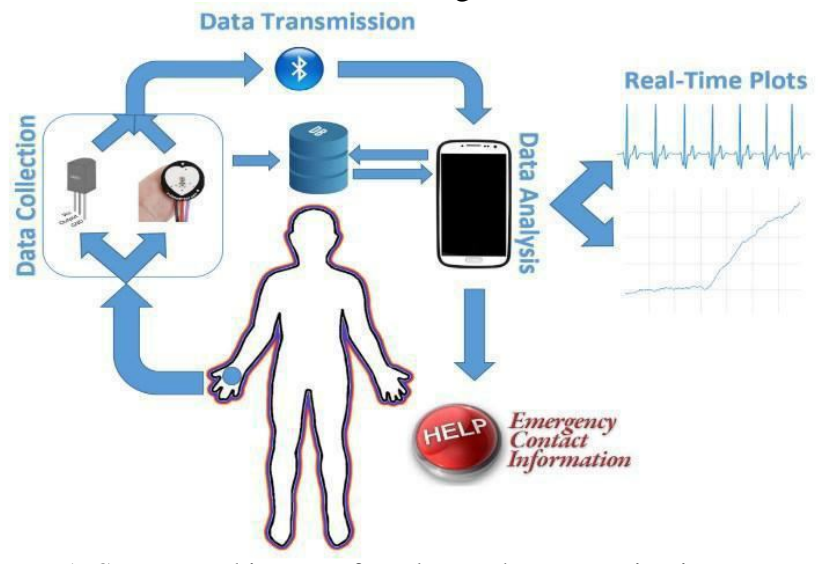

Figure 1. System architecture for Bluetooth communication system

The main drawback of this system is shorter range of data communication.

To overcome the drawbacks, this paper proposes an embedded IoT system for a wireless earlier prediction of cardiac arrest through fog enabled IoT. This system is designed to overcome some of the drawbacks in the existing systems and to potentially yield the good prediction results. The most important aspect of this system is the warning or a notification that allows the user to prevent the emergency injuries before it actually happens. To the best of our knowledge, this system provides faster communication, fault tolerance and the accurate readings by using smartphone and an embedded IoT system for predicting heart related issues. 


\section{ARCHITECTURE OF THE SYSTEM.}

The Main strength of our Proposed Architecture is relying on existing wireless communications, which will provide a low power consumption with maximum functionality through sensor fusion and freedom of movement to users in their physical activity. Here, we have used small and the lightweight devices that are user friendly...

To test the effectiveness of our proposed model, we collected data from ECG, BP, Heart rate and temperature sensors using a Raspberry-pi module and then analyze the data. We used multiple subjects and collected data for the different situations. Data for each subject was collected by the smartphone. The sensors data collection interface is based on the values collected by the sensor that is ECG, BP, Heart rate and body temperature for a period of time. Using Fog computing monitorization, make a decision and store to cloud storage to represent on a Smartphone.

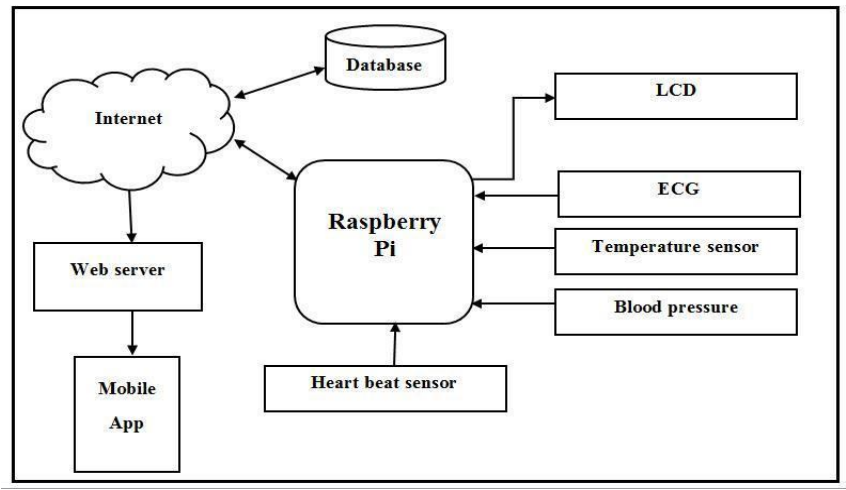

Figure 2. System Architecture

Using raspberry-pi as a main controlling unit, the system will consists of heart beat sensor, BP sensor, temperature sensor and ECG module as shown in Figure 2. And by using Fog computing technique, a decision will be made that the values read by the patient are normal or abnormal and the collected values along with the conditions will be sent to cloud storage and can be retrieved in smartphone by connecting to the internet whenever the user wants to know the condition. If any sensor values exceed the limits then one manual sms notification message will be sent to the user's smartphone, so that he/she can take care of the patient from the critical emergency situations.

\section{HARDWARE}

Here, the IoT system consists of Raspberry-pi, which is an open hardware. With the exception of the primary chip on the raspberry-pi, system on a Chip can run many of the main components of the board such as CPU, graphics, memory, the USB controller, and etc. Many of the projects made with the Raspberry-pi are open and are well documented as well. The things we build here can be modify ourselves. Figure 3, shows how the raspberry-pi will look.

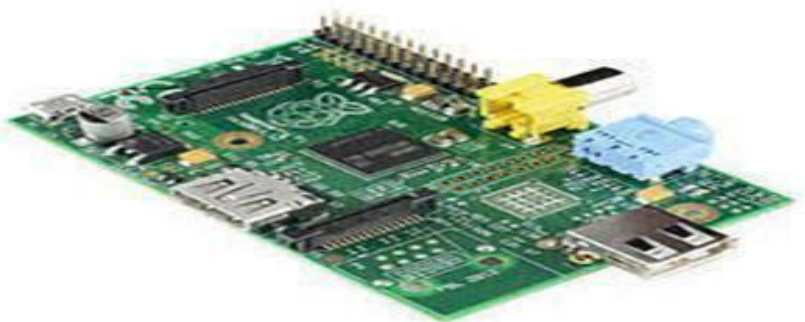

Figure 3. Raspberry pi

The Raspberry-pi is used to operate the system. The Raspberry-pi will be programmed to read the data values form the sensors that are connected to the patient's body. Subsequently it will send that information to the phone as a response to the user's request. It will also manage the Wi-Fi communication by co-ordinating with the Internet facility. With the help of this Wi-Fi communication the data will be sent to the cloud, so that we can collect the sensors data using smart phone.

The sensors that are connected to the raspberry-pi are Heart beat sensor, Temperature sensor, BP sensor and the ECG sensors.

The heartbeat sensor shown in Figure 4.will consists of an light detecting resistor/photodiode and a light emitting diode.

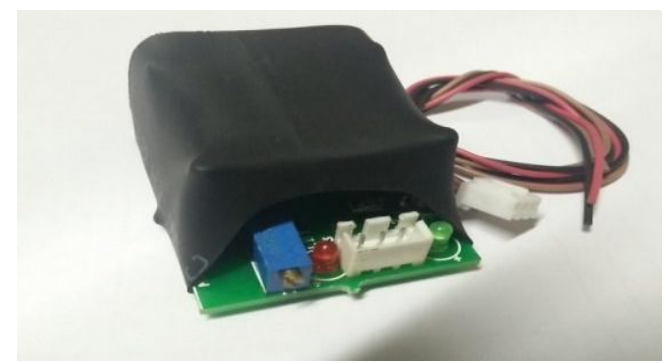

Figure 4. Heart beat sensor

The variation in the flow of blood to the different parts due to change in heart beat pluses. Sensor will transmits or reflects the light whenever the tissue is lighted with the light source. The blood will absorbs some light and light detector will receive the reflected or transmitted light. And the blood volume in the tissue will decide the amount of light absorbed. The heart beat rate will be directly proportional to the electrical signals output form. The electrical signals output form is caused to the pulse rate change in the arterial blood volume.

The LM35 is a temperature sensor that is used to measure the temperature with an electrical output comparative to the temperature in Celsius. This sensor is shown in Figure 5. 


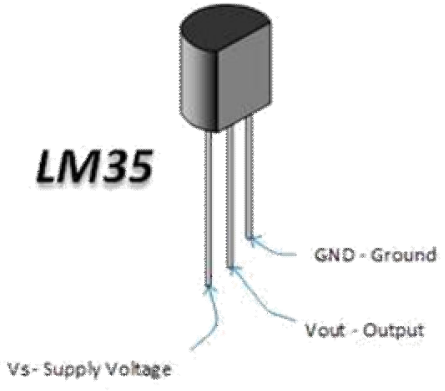

Figure 5. Temperature sensor

It measures the temperature more accurately, maintains an exactness of $+/-0.4^{\circ} \mathrm{C}$ at room temperature and $+/-0.8^{\circ} \mathrm{C}$ at the range of $0^{\circ} \mathrm{C}$ to $+100^{\circ} \mathrm{C}$.

While sensing Blood pressure, we need to put cuff around the patients arm and pump it up, that is by cutting off the blood flow with the pressure exerted by the cuff. As the pressure in the cuff is released, blood will starts flowing again and the blood flow reading will be taken and is sent to the raspberry pi. The BP sensor we used is shown below (Figure 6).

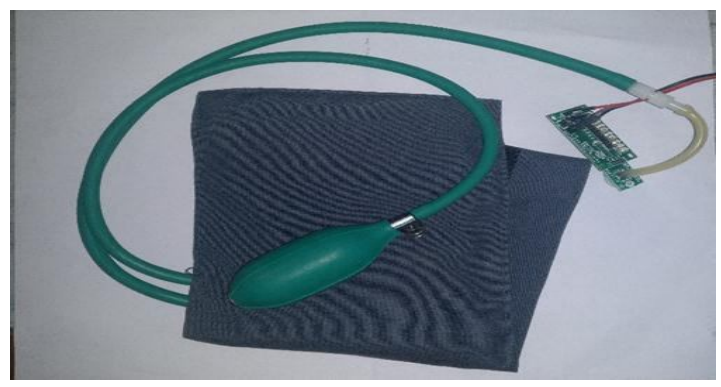

Figure 6. Blood pressure sensor

ECG sensor is shown in Figure 7, It is a used to measure the electrical activity of the heart. This electrical activity can be charted as Electrocardiogram (ECG). Electrocardiography is used to help diagnose various heart conditions.

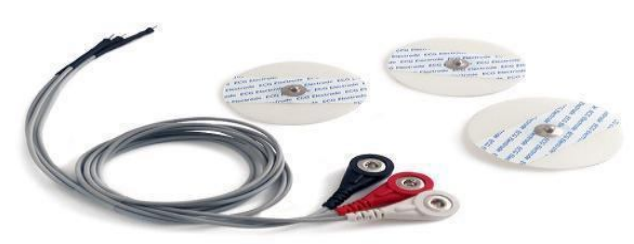

Figure 7. ECG sensors

Electrocardiography (ECG sensor) measures the electrical activity of the heart by placing electrodes on the skin. For the heart to contract and pump the blood, a series of co-ordinated electrical signals will be sent to the heart by the autonomic nervous system. Instead of placing deep inside the body, these signals measures at the skin level effectively by placing two or more electrodes in various positions on the chest and limbs.

\section{EVALUATION OF THE SYSTEM}

To test this system, first we have developed a prototype and estimate its performance. Here we divide this system into 3 sections.

- Experimental setup

- Data collection

- Result analysis

\section{Experimental setup}

To ensure the performance of this proposed model, the sensors values are collected using smart phone. To know the patient's condition, we have collected the respective sensor values from the normal persons and patients, who are suffering from heart diseases.

\section{Data collection}

The values from the sensors are collected by raspberry-pi, and then it will forward to the cloud. The value from the cloud can be collected in the smartphone with the help of internet. And the screenshot of this data collection interface is shown in Figure 8.

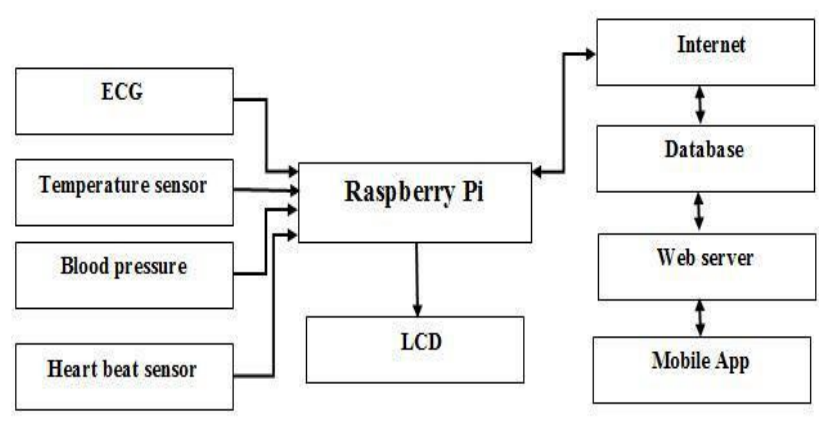

Figure 8. Sensor's data collection interface

We are not able to detect the potential heart attack or cardiac arrest for who are experienced with heart attacks. So we have collected the data from both the healthier person and who are experienced the cardiac arrest to make decisions.

\section{Result analysis}

In this section, the values collected by the sensors are analyzed by using the fog computing technique in raspberry-pi based on threshold values.

By making this analysis, we have a number of advantages like fault detection and isolation, security and privacy and real time services etc. And also the data can be monitored efficiently and can predict the potential failures. 


\section{ALGORITHM}

Here we are making use of prediction algorithm. Once after finishing the extraction of values from the sensors, we compare these values with the analyzed results and apply the prediction algorithm. This process is divided into two major parts

1. First, we make sure that patients body temperature and heart rate is within the normal range by comparing with the threshold values.

\section{INPUT:}

- GET Heartbeat sensor data

- GET human body temperature

- GET blood pressure

- GET Electrocardiography sensor

\section{OUTPUT:}

Read the value of heartbeat sensor, body temperature sensor, $\mathrm{BP}$, electrocardiography sensor showing in LCD and send message through Internet to the smart phone.

\section{Start :}

Read value: temperature sensor value $=\mathrm{TP}[\mathrm{i}]$

Read value: heartbeat sensor value $=\mathrm{HB}[\mathrm{i}]$

Read value: electrocardiography value $=\mathrm{EC}[\mathrm{i}]$

Read value: blood pressure sensor value $=\mathrm{BP}[\mathrm{i}]$

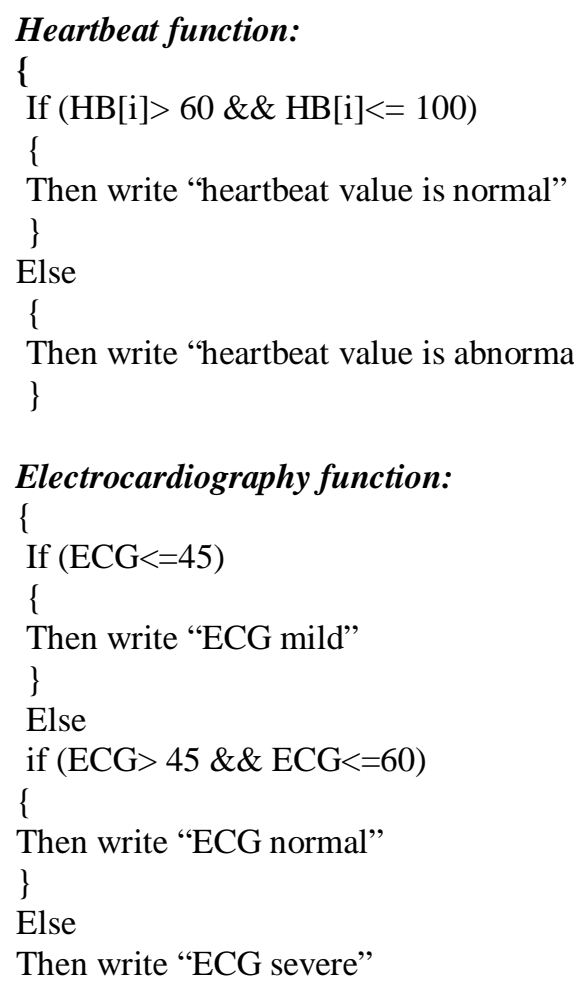

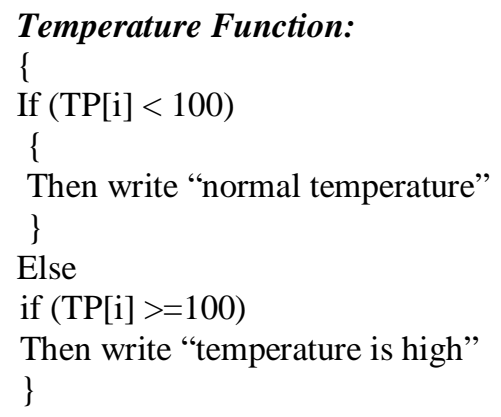

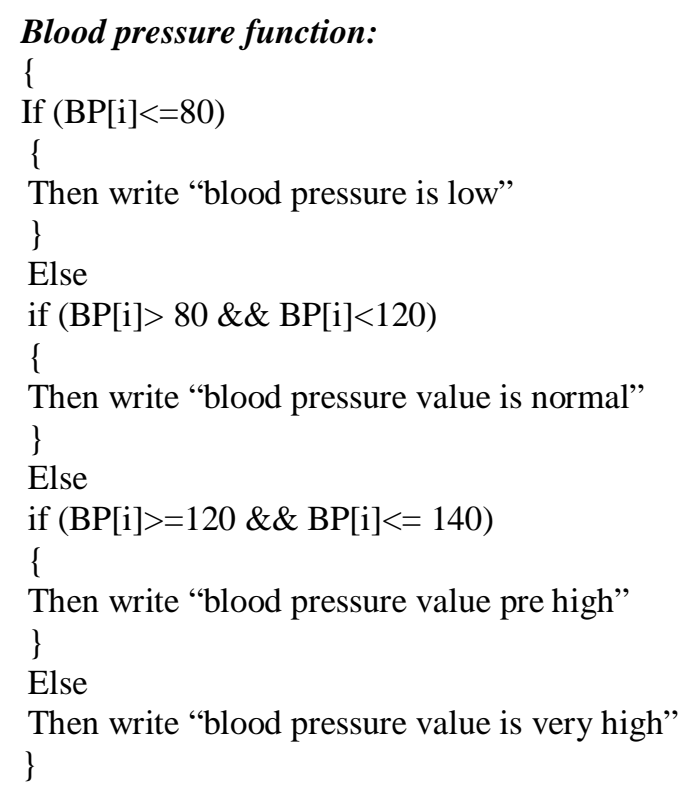

2. Second, if the patient's body temperature and heart rate is not within the normal range then it is abnormal. In this case, the warning message will be sent to the registered user smart phone.

\section{CONCLUSION}

The main goal of this project is to overcome the health issues over the world wide. By implementing the fog computing IoT system, patient's body parameter value can be collected accurately. The cardiac arrest can be predicted earlier and can be diagnosed in the case of emergency, that is by sending an manual sms notification to the registered user smart phone. In future to test the performance and feasibility we plan to collect the data from normal people and who are suffering from the heart problem, so that it helps to monitor and diagnose. 
Deekshitha M R et al., International Journal of Computing, Communications and Networking, 7(2) April - June 2018, 48-53

\section{REFERENCES}

[1] United Nations, Department of Economic and Social Affairs, Population Division, "World Population Ageing 2009," pp. 66-71.

[2] United States, Heart Association and Stroke Association, "Heart Disease and Stroke Statistics - At-a-Glance," 2015

[3] Aaron Smith, "Older Adults and Technology Use", Internet, Science and Technology, PewResearchCenter, April 3, 2014.

[4] J. -V. Lee and Y. -D. C. a. K. T. Chieng, "Smart Elderl Home Monitoring System with an Android Phone," International Journal of Smart Home, vol. 7, pp. 17-32, May 2013.

[5] https://www. sparkfun. Com/search/results? Term=RN+42 [Last Accessed: 22 May, 2017].

[6] ITU-T Global Standards Initiatives Recommendation ITUTY.2060(06/2012)http://www.itu.int/en/ITUT/gsi/iot/Pages/def ault.aspx, [Last Accessed: 22 May, 2017]

[7] O. Vermesan, P Friess, Internet of Things: Converging Technologies for Smart Environments and Integrated Ecosystems, River Publishers Series In Communications, 2013

[8] R. Clarke, Smart Cities and the Internet of Everything: The Foundation for Delivering Next-Generation Citizen Services, Cisco 2013

[9] D. Evans, The Internet of Things How the Next Evolution of the Internet Is Changing Everything, Cisco IBSG, 2011

[10] IEEE Standards Association (IEEE-SA), Internet of Things (IoT) Ecosystem Study, IEEE 2015

[11] [11]Qardiocore,https://www.getqardio.com/qardiocoreweara ble-ecgekg-monitor-iphone/, [ Lat Accessed: 22 May 2017]

[12] J. Penney, Choosing an IoT security provider, http://info.deviceauthority.com/blog-da/choosing-an-iot-secur ityprovider, 2016

[13] B. Djamaa , R. Witty, An efficient service discovery protocol for 6LoWPANs, Science and Information Conference (SAI), IEEE 2013

[14] M. Maksimoviæ, V. Vujoviæ, B. Perišic, "A Custom Internet of Things Healthcare System", Tenth Iberian Conference on Information Systems and Technologies (CISTI), 2015

https://doi.org/10.1109/CISTI.2015.7170415

[15] United Nations, Department of Economic and Social
Affairs, Population Division, "World Population Ageing 2009," pp. 66-71.

[16] United States, Heart Association and Stroke Association, "Heart Disease and Stroke Statistics - At-a-Glance," 2015

[17] S. Aaron, "Older Adults and Technology Use", Internet, Science and Technology, PewResearchCenter, April 3, 2014

\section{AUTHORS BIOGRAPHY}

Prof. Anusha K L: Assistant Professor of Department of Computer Science \& Engineering, East West Institute of Technology (VTU), Bangalore. Qualification: B.E, M.Tech. Her areas of interest are Image Processing, Software Defined Networking Engineering, Internet of Things, Cloud Computing.

Deekshitha M R: Pursuing B.E in CSE, EWIT (VTU), Bangalore. Her areas of interest are Database(sql), Computer Networks, Software Engineering, Manual testing, Internet of Things, etc.

Karthik S: Pursuing B.E in CSE, EWIT (VTU), Bangalore. His areas of interest are Databases(sql), Computer Networks, C programming, Manual testing, Software Engineering, Internet of Things etc.

Lavanya G: Pursuing B.E in CSE, EWIT (VTU), Bengaluru. Her areas of interest are Databases, Computer Networks, Internet of Things, Programming the Web, Software Engineering, Java language, etc.

Mohan Kumar D: Pursuing B.E in CSE, EWIT (VTU), Bangalore. His areas of interest are Databases, Computer Networks, Software Engineering, Internet of Things, Programming the Web, etc. 